\title{
Mapping of TMPRSS2-ERG fusions in the context of multi-focal prostate cancer
}

\author{
Bungo Furusato ${ }^{1,2, *}$, Chun-Ling Gao ${ }^{1, *}$, Lakshmi Ravindranath ${ }^{1}$, Yongmei Chen ${ }^{1}$, \\ Jennifer Cullen ${ }^{1}$, David G McLeod ${ }^{1,3}$, Albert Dobi ${ }^{1}$, Shiv Srivastava ${ }^{1}$, Gyorgy Petrovics ${ }^{1}$ \\ and Isabell A Sesterhenn ${ }^{2}$
}

\begin{abstract}
${ }^{1}$ Department of Surgery, United States Military Cancer Institute, Center for Prostate Disease Research, Uniformed Service University of the Health Science, Bethesda, MD, USA; ${ }^{2}$ Department of Genitourinary Pathology, Armed Forces Institute of Pathology, Washington, DC, USA and ${ }^{3}$ Urology Service, Walter Reed Army Medical Center, Washington, DC, USA
\end{abstract}

\begin{abstract}
TMPRSS2-ERG gene fusion leading to the androgenic induction of the ERG proto-oncogene expression is a highly prevalent oncogenic alteration in prostate tumor cells. Prostate cancer is a multi-focal disease, and the origins as well as biological contribution of multiple cancer foci remain unclear with respect to prostate cancer onset or progression. To assess the role of TMPRSS2-ERG alteration in prostate cancer onset and/or progression, we have evaluated the status of fusion transcripts in benign glands, prostatic intraepithelial neoplasia (PIN) and multiple cancer foci of each prostate. Quantitative expression of TMPRSS2-ERG fusion type $A$ and $C$ transcripts was analyzed in benign, tumor and PIN areas, selected from whole-mount radical prostatectomy slides. TMPRSS2-ERG expression was correlated with clinicopathological features. Overall, 30 of $45(67 \%)$ patients exhibited TMPRSS2-ERG fusion transcripts in at least one tumor focus. Of 80 tumor foci analyzed, 39 had TMPRSS2-ERG fusion (type A only: 30, type C only: 2, both types A and C: 7), with predominant detection of the TMPRSS2-ERG fusion type $A(27 / 30,90 \%)$ in the index tumors. Of 14 PIN lesions, 2 were positive for type A fusion. Frequent presence of the TMPRSS2-ERG in index tumors suggests critical roles of ERG alterations in the onset and progression of a large subset of prostate cancer. However, heterogeneity of the TMPRSS2-ERG detection in the context of multiple cancer foci and its frequency in PIN also support the role of other genomic alterations in the origins of prostate cancer.
\end{abstract}

Modern Pathology (2008) 21, 67-75; doi:10.1038/modpathol.3800981; published online 7 December 2007

Keywords: multi-focal; prostate cancer; TMPRSS2; ERG; gene fusion

Prostate cancer has been recognized as a multi-focal disease. ${ }^{1-3}$

Currently, in our series of over 1150 radical prostatectomies most specimens contain two or more independent tumors. It is still unclear whether each of these cancer foci contributes to patient's prognosis after the radical prostatectomy. We and others found that the larger tumors typically contain poorly differentiated foci. ${ }^{1,2}$ Stamey et $a l^{4}$ reported that percent Gleason grade $4 / 5$, cancer volume of the largest tumor, positive lymph node findings and intraprostatic vascular invasion have been independently associated with prostate cancer progression.

Correspondence: Dr IA Sesterhenn, MD, Department of Genitourinary Pathology, Armed Forces Institute of Pathology, 6825 16th Street NW, Washington, DC 20307, USA.

E-mail: sesterhe@afip.osd.mil

*These authors contributed equally to this work.

Received 08 October 2007; revised 24 October 2007; accepted 31 October 2007; published online 7 December 2007
Intertumoral as well as intratumoral heterogeneity with respect to genomic alterations has also been noted in multiple foci. ${ }^{5}$ The biological significance of multiple independent foci of prostate cancer, histological grade heterogeneity within each tumor focus and difference in the anatomical location of the tumor are still unclear.

Recent reports of prevalent chromosomal rearrangements/translocations, leading to the activation of ETS transcription factors (predominantly ERG) through androgen receptor-inducible TMPRSS2 gene promoter, ${ }^{6}$ underscore the critical role of $E R G$ overexpression in prostate cancer. ${ }^{7}$ Subsequent reports, ${ }^{8-12}$ including our study, ${ }^{13}$ have shown high frequency of TMPRSS2-ERG fusion and/or overexpression in clinically localized prostate cancers.

TMPRSS2 encodes an androgen-dependent transmembrane serine protease, which is strongly expressed in both normal tissue and prostate cancer. ETS transcription factors regulate the expression of genes with important cancer-relevant biological 
processes, such as cell growth, differentiation, and transformation. ${ }^{14}$ TMPRSS2-ERG fusion may lead to prostate cancer cell-associated androgenic regulation of $E R G^{6}$ in prostate cancer cells. However, the biologic role of ERG overexpression in prostate cancer development and progression remains to be defined. Since TMPRSS2-ERG fusions lead to frequent alterations $(>60 \%)$ of the ERG protooncogene in early-stage prostate cancer, their evaluation in preneoplastic cells as well as multi-focal lesions from the same patient has potential to define its role in the prostate cancer onset, progression, and heterogeneity. This study analyzes TMPRSS2-ERG alterations in prostatic intraepithelial neoplasia (PIN) and multi-focal cancers sampled from wholemount prostate glands of prostate cancer patients.

\section{Materials and methods}

\section{Cell Culture}

Human prostate cancer cell line VCaP (cat. no. CRL2876; American Type Culture Collection, Rockville, MD, USA) with known TMPRSS2-ERG fusion was grown in Dulbecco's modified Eagle's medium containing $4 \mathrm{mM}$ L-glutamine, $1.5 \mathrm{~g} / \mathrm{l}$ sodium bicarbonate, $4.5 \mathrm{~g} / \mathrm{l}$ glucose, and $10 \%$ fetal bovine serum. VCaP cells were used as positive control for TMPRSS2-ERG fusion expression and were also used for determining the sensitivity of detecting TMPRSS2-ERG fusion.

\section{TMPRSS2-ERG Fusion Measurements in Formalin-Fixed Paraffin-Embedded VCaP Cells}

To develop a set of positive controls for TMPRSS2$E R G$ fusion assessment in tissue sections, $\mathrm{VCaP}$ cell pellets were fixed with formalin and processed as paraffin blocks. A paraffin block containing VCaP cells was sectioned and utilized for checking the sensitivity and reproducibility of TMPRSS2-ERG fusion in formalin-fixed paraffin-embedded (FFPE) material. We determined that a minimum of $100 \mathrm{pg}$ of VCaP RNA was needed for a reproducible realtime RT-PCR-based TMPRSS2-ERG fusion detection assay. PCR primers and PCR conditions are given in the Quantitative real-time RT-PCR section below.

\section{Whole-Mount FFPE-Prostate Specimens and Correlation of TMPRSS2-ERG Expression with Clinicopathological Data}

This study was performed under IRB-approved protocols (WU no. 04-28030, and WU no. 04-287198k). Prostatectomy specimens from patients treated at Walter Reed Army Medical Center were sectioned and embedded as whole mounts at the Armed Forces Institute of Pathology. After surgery, radical prostatectomy specimens were immediately fixed in $10 \%$ buffered formalin and embedded as whole mounts in paraffin. Each prostate was sectioned at $0.22 \mathrm{~cm}$ intervals in a transverse plane perpendicular to the long axis of the posterior surface of the prostate and completely embedded as whole mounts. The volume of each tumor was calculated in three dimensions (apex to base, right to left, and anterior to posterior) using the largest dimension in each direction. The volume did not include the shrinkage factor to correct for fixation and embedding. The tumor volumes of the measurable foci, from the largest (T1) to the smallest (T4) were recorded as T1-T4. The tumor dimension was not corrected for the actual tumor shape of the crosssection and, therefore, does not represent the true size of the tumor in cross-sectional area.

One $4-\mu \mathrm{m}$ whole-mount slide was stained with hematoxylin and eosin, and used for a control. The adjacent two $6-\mu \mathrm{m}$ sections were used for tissue dissection. Quantitative expression of the TMPRSS2-ERG was analyzed for a relationship with patient age at the time of surgery, race, clinical stage, preoperative PSA, prostatectomy pathology data (specimen size, tumor volume, grade, stage, and surgical margin status), and follow-up PSA data.

\section{Definition of Non-Malignant Tissue and PIN}

Epithelium was classified as histologically nonmalignant when glands contained two cell layers: the luminal cells with inconspicuous nucleoli and basal cells. Native glands were classified as PIN, when the secretory cells showed nuclear anaplasia and basal cells were detectable. ${ }^{15}$ PIN adjacent to carcinoma (PIN located within $2 \mathrm{~mm}$ distance from the edge of tumor foci) and PIN distant from carcinoma (PIN located more than $2 \mathrm{~mm}$ distant from edge of tumor foci) were also evaluated for TMPRSS2-ERG fusion.

\section{Tissue Micro-Dissections}

Unstained 6- $\mu \mathrm{m}$-thick tissue sections on glass slides were deparaffinized once with citrasolve buffer (cat. no. 22-143-975; Fisher Scientific, Pittsburgh, PA, USA) for $10 \mathrm{~min}$, rinsed twice with $100 \%$ ethanol for $10 \mathrm{~min}$, and air-dried. Specific histological fields of interest were selected (and ink marked) under the microscope by a genitourinary pathologist. Microdissection was performed using a pipette with an ultrafine pointed tip (cat. no. 2149p; Molecular BioProducts, San Diego, CA, USA). The histological area of interest on the tissue section was gently scraped with the pipette tip and RNA extraction buffer (total $250 \mu \mathrm{l}$ RNA lysis buffer; $20 \mathrm{mM}$ Tris (pH 7.4), $20 \mathrm{mM}$ EDTA, $1 \%$ SDS, and $1 \mathrm{mg} / \mathrm{ml}$ proteinase K) until the selected cells became detached from the tissue section. Samples were transferred to Eppendorf tube and incubated overnight in $55^{\circ} \mathrm{C}$ water bath. After incubation, $750 \mu \mathrm{l}$ TRIzol (cat. no. 15596026; Invitrogen, Carlsbad, CA, USA) was added into 
the lysis buffer to extract RNA according to the manufacturer's recommendations. RNA was treated by DNase I (cat. no. 18068; Invitrogen) for $15 \mathrm{~min}$ at room temperature. RNA concentration was measured by Agilent 2100 Bioanalyzer (Agilent Technologies, Santa Clara, CA, USA).

\section{Quantitative Real-Time RT-PCR}

Total RNA (100 ng) was reverse transcribed to cDNA using Omniscript reverse transcriptase (cat. no. 20513; Qiagen Technology, Germantown, MD, USA) and random hexamer primers in a volume of $20 \mu \mathrm{l}$. For each PCR, cDNA equivalent to $22.5 \mathrm{ng}$ of total RNA was used in a final volume of $30 \mu \mathrm{l}$. Quantitative real-time PCR was performed using SYBR green master mix (cat. no. 4309155; Applied Biosystems, Foster City, CA, USA) in ABI PRISM 7700 sequence analyzer (Applied Biosystems). Each patient sample was analyzed in duplicate. Thermal cycling conditions were as follows: initial one-step incubation at $50^{\circ} \mathrm{C}$ for $2 \mathrm{~min}$, followed by a single denaturation step at $95^{\circ} \mathrm{C}$ for $10 \mathrm{~min}$. Two-step PCR was then carried out for 45 cycles with $95^{\circ} \mathrm{C}$ denaturation for $15 \mathrm{~s}$, followed by annealing/extension at $60^{\circ} \mathrm{C}$ for $60 \mathrm{~s}$. Fluorescence intensity measurements were made at the end of each extension phase. The following PCR primers were used in the study: TMPRSS2-ERG fusion A sense primer: CGCGGCAGGAAGCCTTAT (located in TMPRSS2 exon 1), antisense primer: CGCGGTCATCTCTGTC TAGC (ERG exon 4); TMPRSS2-ERG fusion C sense primer: AGCGCGGCAGGAACTCT (TMPRSS2 exon 1), antisense primer: CCATGTAGCTGCCGTAGTT CATC (ERG exon 5); PSA sense primer: CCCACTG CATCAGGAACAAA, antisense primer: GAGCGGGT GTGGGAAGCT; $\beta$-2-microglobulin sense primer: TG ACTTTGTCACAGCCCAAGATA, antisense primer: ATTCCAAATGCGGCATATTC. A 9 pmol portion of each primer was used in the PCRs. RT-PCR for $P S A$ and $\beta$-2-microglobulin served as RNA quality control. Each PCR product was independently confirmed for expected size by electrophoresis on the TBE-agarose gel (1.5\%). Schematic diagrams of TMPRSS2-ERG fusion variants (types $\mathrm{A}$ and $\mathrm{C}$ ) and sensitivity assay of quantitative RT-PCR for TMPRSS2-ERG fusion A using RNA from FFPE VCaP cells are shown in Figure 1.

\section{Statistical Analysis}

Clinicopathological parameters, including age at time of surgery, preoperative PSA level, race, clinical stage, pathological stage, Gleason score, surgical margin status, tumor volume and PSA recurrence were compared with the presence of TMPRSS2-ERG fusion. For this analysis, Student's $t$-test and Fisher exact test were used. To determine whether the TMPRSS2-ERG fusion is a prognostic factor for cancer progression, we performed survival analysis, using PSA recurrence as primary end point. This was defined as two consecutive postoperative PSA $\geq 0.2 \mathrm{ng} / \mathrm{ml}$. PSA recurrence-free survival curves were compared between patients with and without expression of TMPRSS-ERG fusion using Kaplan-Meier unadjusted survival analysis method. Univariate Cox proportional hazard analysis was carried out to examine odds of PSA recurrence over time for all clinicopathological parameters, as well as TMPRSS2-ERG fusion. Hazard ratio and corresponding confidence interval and $P$-values are reported. $P$-value $<0.05$ was adopted as statistically significant. The SAS version 9.1 was used for all data analysis.

\section{Results}

The detailed topographical data of TMPRSS2-ERG fusion in multiple tumor foci of each patient are summarized in Table 1. Representative wholemount prostate sections of six patients with TMPRSS2-ERG fusion transcript status are also shown in Figure 2. Overall, 30 of 45 patients (67\%) exhibited TMPRSS2-ERG fusion transcripts in at least one tumor focus. Heterogeneity of fusion transcripts in same patient specimen were apparent (Figure 2). Of 80 tumor foci analyzed, 39 (49\%) had TMPRSS2-ERG fusion. In these 39 fusion-positive tumor foci, 30 demonstrated the TMPRSS2-ERG fusion type A only, 2 showed TMPRSS2-ERG fusion type $\mathrm{C}$ only, and 7 had both types $\mathrm{A}$ and $\mathrm{C}$.

In PIN lesions, overall 2 of 14 foci were positive for fusion type A. In 5 of 14 cases, PIN was located adjacent to tumor (PIN located within $2 \mathrm{~mm}$ distance from edge of tumor foci) and 1 of these 5 foci was positive for TMPRSS2-ERG fusion type A. Among the PIN lesions distantly located (PIN located more than $2 \mathrm{~mm}$ distant from edge of tumor foci), 1 of 9 foci had TMPRSS2-ERG fusion type A.

Intriguingly, 2 of 45 RNA specimens from the benign prostatic glands also showed TMPRSS2-ERG fusion type A. These two cases were subjected to repeated pathological examination. However, based on morphology we could not identify malignancy in the areas sampled.

To determine whether the presence of TMPRSS2$E R G$ fusion type A in prostate cancer tissue was associated with disease progression, we compared PSA recurrence status between patients with or without fusion (Table 2). Among the 28 patients who had TMPRSS2-ERG fusion type A-positive tumors, 3 of 28 experienced PSA recurrence, whereas 4 of the 17 patients who had tumors without fusion transcript experienced PSA recurrence. There was no statistical difference between patients who had tumors with and without TMPRSS2-ERG fusion type A and PSA recurrence $(P=0.3986)$. This was also confirmed by Kaplan-Meier unadjusted survival analysis, which did not show significance $(P=0.42)$. 


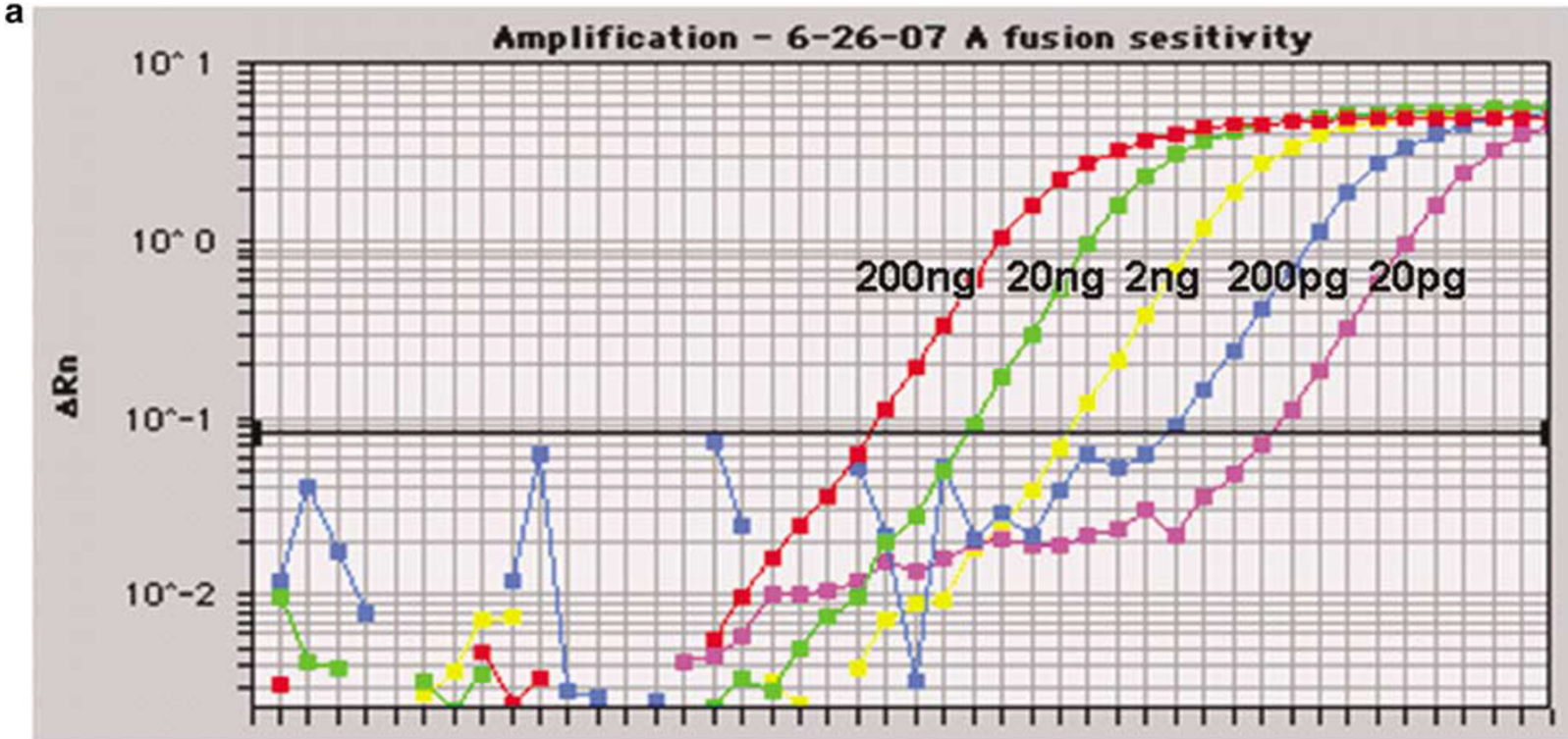

b

VCaP RNA

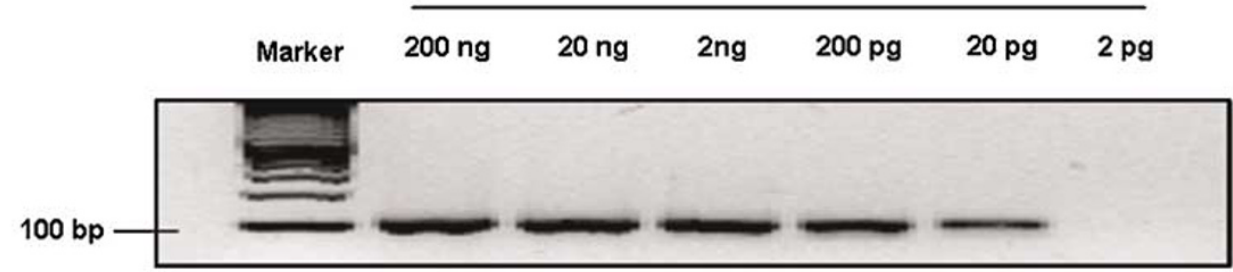

C

TMPRSS2-ERG gene fusion types A and C

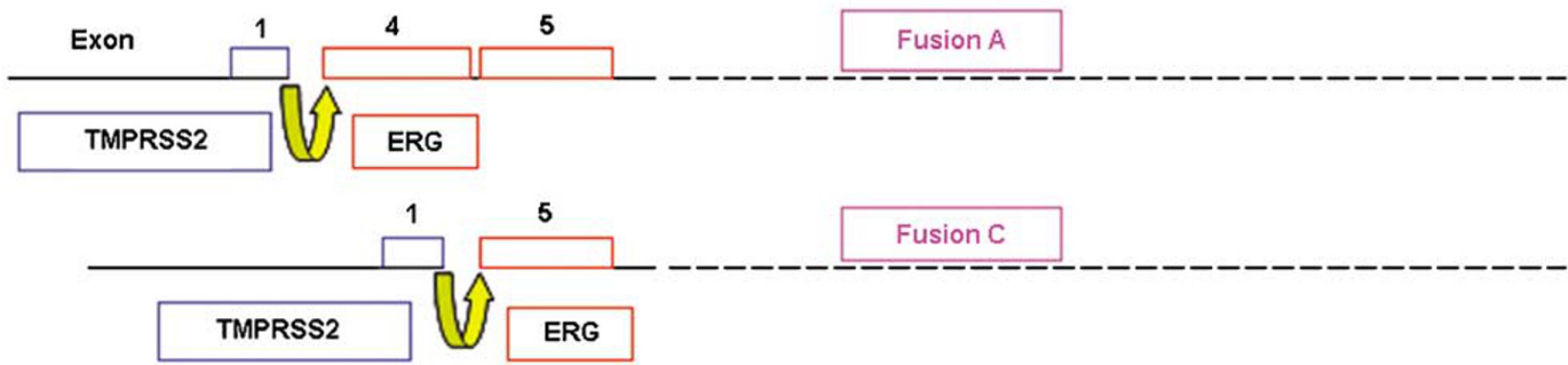

Figure 1 Sensitivity assay for TMPRSS2-ERG fusion and schematic representation of TMPRSS2-ERG fusion A and C transcripts. Serial dilutions of VCaP RNA were assayed for TMPRSS2-ERG fusion type A expression by quantitative RT-PCR. (a) Quantitative real-time RTPCR plot. (b) Gel electrophoresis of the PCR products. (c) Exons are represented by numbered boxes. The arrow symbolizes the fusion.

We also examined whether the presence of TMPRSS2-ERG fusion transcripts, especially TMPRSS2-ERG fusion type A, correlated with other prognostic variables. All the parameters, including age at diagnosis, race, preoperative PSA, clinical stage, pathological stage, Gleason score, surgical margin, prostate size, and index tumor volume, did not correlate with the presence of TMPRSS2-ERG fusion type A (Table 2).

In a univariate Cox model, there was no relationship of the TMPRSS2-ERG fusion type A with various clinicopathologic variables; only Gleason score $(P=0.0405)$ and index tumor volume $(P=0.0132)$ were significant predictors for PSA recurrence (Table 3).

In addition, we examined whether TMPRSS2$E R G$ fusion type A was frequently present in the index tumor by using Fisher exact test. For this analysis, a total of 27 prostatectomies were available, which contained at least both $\mathrm{T} 1$ (index or largest) and T2 (second largest) tumor in a given whole-mount section. TMPRSS2-ERG fusion type A was prevalent in 19 of 27 T1 compared to 8 of 27 T2. However, due to the small sample size, it was not statistically significant $(P=0.36)$. 
Table 1 Comparisons of TMPRSS2-ERG fusion pattern in separate tumors and non-malignant area from the same prostate

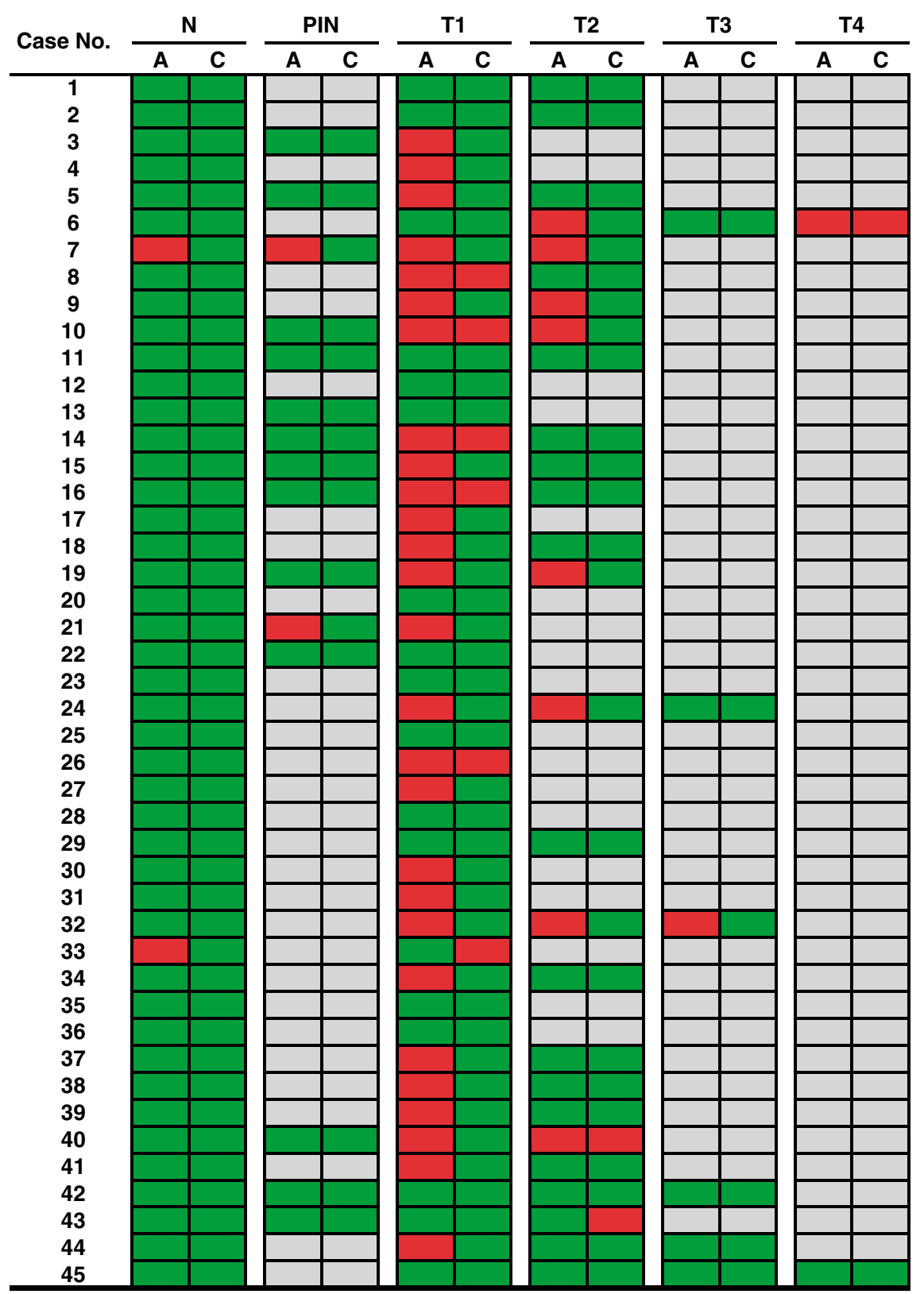

Tumors $\mathrm{T} 1, \mathrm{~T} 2, \mathrm{~T} 3$, and $\mathrm{T} 4$ designate separate tumors in the same prostate. Red = positive for TMPRSS2-ERG fusion; green $=$ negative for TMPRSS2-ERG fusion; gray = not evaluable; $\mathrm{A}=$ TMPRSS2-ERG fusion type $\mathrm{A} ; \mathrm{C}=$ TMPRSS2-ERG fusion type $\mathrm{C}$; $\mathrm{N}=$ non-malignant.

\section{Discussion}

Most prostates contain multi-focal tumors. ${ }^{1,16-19}$ The heterogeneity of prostate cancer in multi-focal tumor context has also been well documented. The question arises whether these multi-focal tumors in the same prostate are of independent origin. To address this question, we analyzed the common genomic alterations of the TMPRSS2-ERG, recently described by several groups in clinically localized prostate cancer. ${ }^{6,20-41}$

In the study by Gao et al, ${ }^{13}$ at least one tumor focus of $67 \%$ of patients exhibited TMPRSS2-ERG fusion, of which is similar to our recent finding of $62 \%$ in laser capture microdissected OCT-embedded frozen specimens. We found that half of the specimens collected from the index tumor contained this molecular alteration. 


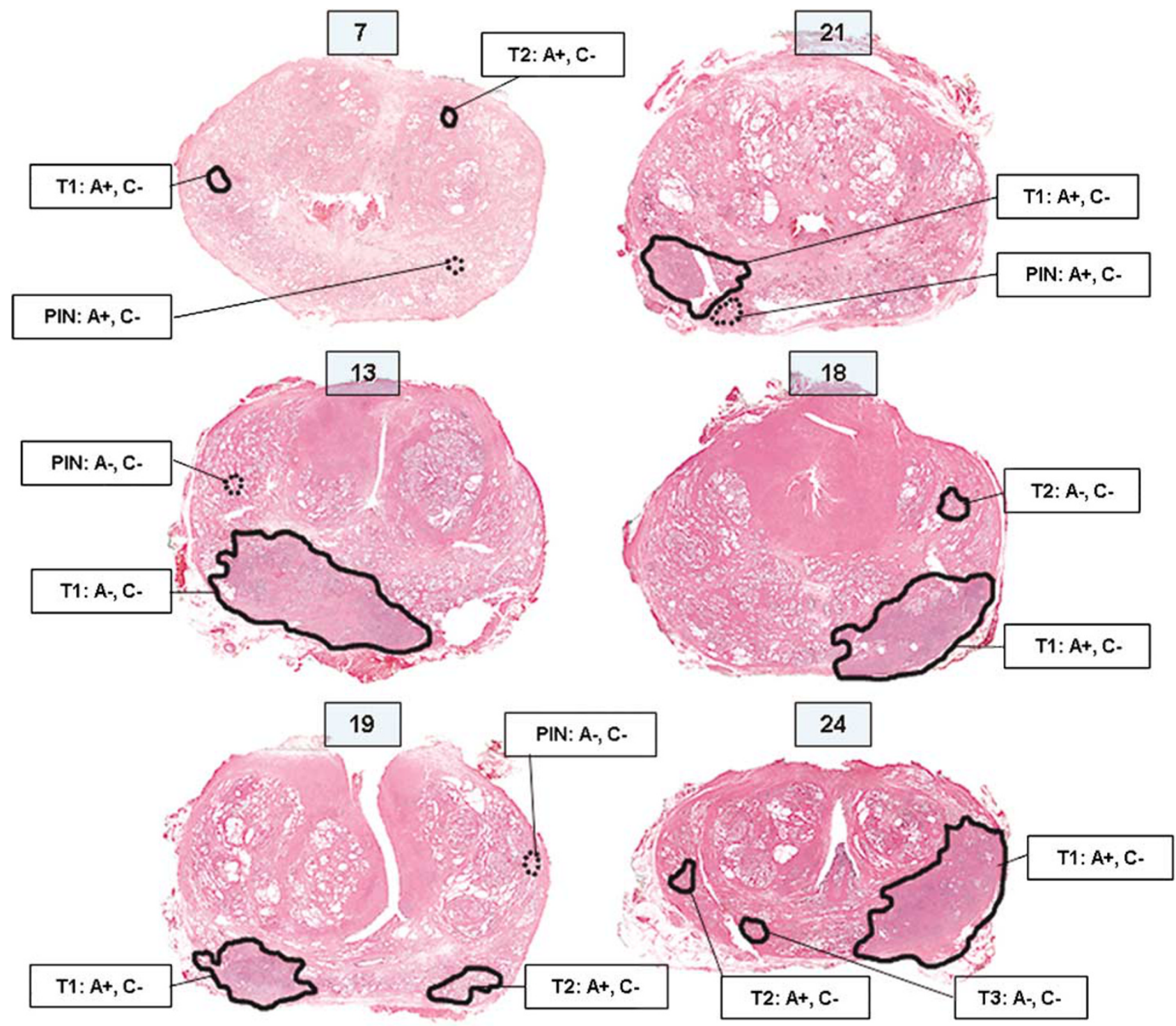

Figure 2 Topographical data of TMPRSS2-ERG fusion from six patients. Tumors T1, T2, and T3 designate separate tumors in the same prostate. PIN = prostatic intraepithelial neoplasia; $+=$ positive for TMPRSS2-ERG fusion; $-=$ negative for TMPRSS2-ERG fusion; $\mathrm{A}=$ TMPRSS2-ERG fusion type A; C = TMPRSS2-ERG fusion type C.

This study highlights the frequent presence of the TMPRSS2-ERG fusion transcripts in index tumors and suggests a critical role of $E R G$ alterations in the onset and/or progression of a large subset of prostate cancer. TMPRSS2-ERG heterogeneity in separate tumor foci of the same patient further reinforces the concept of independent origin of multiple tumors, which had been addressed. . $^{1,43}$

There was no statistical difference between patients who had tumors with and without TMPRSS2-ERG fusion type A and PSA recurrence $(P=0.3986)$. The current data based on 45 patients show a similar trend as in our previous study based on frozen specimens. ${ }^{13}$ However, the lack of prognostic significance of TMPRSS-ERG status may have been masked by the limited sample number.
Of 45 non-malignant samples, 2 showed TMPRSS2$E R G$ fusion. When these two cases were subjected to a repeated pathological examination, we could not identify PIN or carcinoma but some morphologically benign glands, some of which showed $\alpha$-methylacylCoA racemase expression in luminal cells by immunohistochemistry. Previously, Leav et $a l^{44}$ found that by using $\alpha$-methylacyl-CoA racemase it is possible to define areas of transition from benign glands to malignant glands. Virtually, all of the TMPRSS2-ERG fusion reports show the gene fusion or the fusion transcripts in malignant glands. ${ }^{6,21-40}$ Clark et $a t^{41}$ detected several patterns of gene TMPRSS2-ERG fusion transcripts, including type $\mathrm{A}$ in 2 of $31(6 \%)$ 'benign prostatic hyperplasia' and in 8 of 17 (43\%) 'non-malignant specimens.' It is conceivable that 
Table 2 Clinicopathologic correlation between TMPRSS2-ERG fusion-positive and -negative cases

\begin{tabular}{|c|c|c|c|}
\hline Parameter & TMPRSS2-ERG fusion negative & TMPRSS2-ERG fusion positive & $\mathrm{P}$-value \\
\hline Age at surgery (years) & $59.1 \pm 7.8$ & $57.4 \pm 8.8$ & 0.5068 \\
\hline Prostate size $\left(\mathrm{cm}^{3}\right)$ & $52.2 \pm 38.7$ & $43.0 \pm 21.6$ & $0.6184^{\mathrm{a}}$ \\
\hline Index tumor volume $\left(\mathrm{cm}^{3}\right)$ & $6.0 \pm 9.4$ & $4.5 \pm 3.9$ & $0.7963^{\mathrm{a}}$ \\
\hline Race & & & 1 \\
\hline African American & $3(42.9 \%)$ & $4(57.1 \%)$ & \\
\hline Caucasian & $14(36.8 \%)$ & $24(63.2 \%)$ & \\
\hline$P S A(n g / m l)$ & & & 0.7401 \\
\hline$<4.0$ & $6(40.0 \%)$ & $9(60.0 \%)$ & \\
\hline $4.0-10$ & $7(36.8 \%)$ & $12(63.2 \%)$ & \\
\hline$>10$ & $3(60.0 \%)$ & $2(40.0 \%)$ & \\
\hline Clinical stage & & & 0.731 \\
\hline $\mathrm{T} 1$ & $10(40.0 \%)$ & $15(60.0 \%)$ & \\
\hline $\mathrm{T} 2-3$ & $7(35.0 \%)$ & $13(65.0 \%)$ & \\
\hline Pathological stage & & & 0.283 \\
\hline pT2 & $8(47.1 \%)$ & $9(52.9 \%)$ & \\
\hline pT3 & $7(30.4 \%)$ & $16(69.6 \%)$ & \\
\hline Gleason score & & & 1 \\
\hline $2-6$ & $8(38.1 \%)$ & $13(61.9 \%)$ & \\
\hline 7 & $7(41.2 \%)$ & $10(58.8 \%)$ & \\
\hline $8-10$ & $2(33.3 \%)$ & $4(66.71 \%)$ & \\
\hline Surgical margin status & & & 0.277 \\
\hline Negative & $13(43.3 \%)$ & $17(56.7 \%)$ & \\
\hline Positive & $4(26.7 \%)$ & $11(73.3 \%)$ & \\
\hline PSA recurrence status & & & 0.3986 \\
\hline No & $13(34.2 \%)$ & $25(65.8 \%)$ & \\
\hline Yes & $4(57.1 \%)$ & $3(42.9 \%)$ & \\
\hline
\end{tabular}

${ }^{\mathrm{a}} P$-value comes from $t$-test based on log transformation.

Table 3 Univariate Cox model for biochemical-free survival after surgery

\begin{tabular}{|c|c|c|c|}
\hline Variables & Hazard ratio & 95\% confidence interval & P-value \\
\hline Age at surgery (years) & 1.134 & $0.352-3.650$ & 0.8334 \\
\hline Race (Caucasian vs African American) ${ }^{\mathrm{a}}$ & - & - & - \\
\hline Clinical stage (T2-3 vs T1) & 2.12 & $0.388-11.578$ & 0.3857 \\
\hline Preoperative PSA (ng/ml) & & & 0.4554 \\
\hline $4-10$ vs $<4$ & 4.048 & $0.452-32.274$ & 0.2114 \\
\hline$>10$ vs $<4$ & 3.442 & $0.215-55.117$ & 0.3823 \\
\hline Pathologic stage (T3 vs T2) & 3.59 & $0.401-32.156$ & 0.2533 \\
\hline Gleason score & & & 0.0405 \\
\hline 7 vs 6 & 3.832 & $0.347-42.297$ & 0.2729 \\
\hline $8-10$ vs 6 & 16.075 & $1.651-156.558$ & 0.0168 \\
\hline Surgical margin (positive vs negative) & 5.239 & $0.957-28.671$ & 0.0562 \\
\hline Index tumor volume $\left(\mathrm{cm}^{3}\right)$ & 1.088 & $1.018-1.164$ & 0.0132 \\
\hline TMPRSS2-ERG fusion (positive vs negative) & 0.508 & $0.102-2.518$ & 0.4071 \\
\hline
\end{tabular}

${ }^{\mathrm{a}}$ There is no PSA recurrence in African-American men.

TMPRSS2-ERG fusion type A-positive benign glands may have the potential to develop into PIN or carcinoma. However, this hypothesis has to be further evaluated in a large cohort study.
We also found that a subset of PIN lesions (2 of 14 foci) showed TMPRSS2-ERG fusion type A. These PIN lesions with detectable TMPRSS2-ERG fusion may undergo further genetic changes to develop 
prostate cancer. Linkage of PIN (high-grade PIN) and cancer has been well documented. ${ }^{45}$

\section{Conclusions}

Information regarding the origin of multiple tumors in prostate cancer is critical to our understanding of the pathobiology and natural history of this disease. In this regard, prevalent oncogenic alterations of $E R G$ due to TMPRSS2-ERG fusion in early-stage prostate cancer provide a break through observation to investigate the role of this potentially causal prostate cancer-specific genomic alteration in the origins of multi-focal prostate cancer. Our data show that half of the malignant areas in prostates harbor TMPRSS2-ERG fusions and apparent heterogeneity of TMPRSS2-ERG supports the independent origins of multi-focal tumors in the same prostate. Based on limited data, we suggest that foci of benign glands or PIN with TMPRSS2-ERG fusion type A may give rise to carcinoma. Thus, detection of benign glands with TMPRSS2-ERG fusion in biopsy or TURP specimen might suggest that these patients should be closely monitored.

\section{Acknowledgement}

We thank Ms Denise Young for preparation of the prostate samples. This research was supported by the Center for Prostate Disease Research Program through the Henry M Jackson Foundation for the Advancement of Military Medicine under contract number HU001-04-C-1502 and by the NIH Grant RO1 DK065977 (GP and SS).

\section{Conflict of interest}

The authors indicated no potential conflict of interest. The views expressed in this article are those of the authors and do not reflect the official policy of the Department of the Army, Department of Defense, or the US Government.

\section{References}

1 Byar DP, Mostofi FK. Carcinoma of the prostate: prognostic evaluation of certain pathologic features in 208 radical prostatectomies. Examined by the stepsection technique. Cancer 1972;30:5-13.

2 McNeal JE, Price HM, Redwine EA, et al. Stage A versus stage $B$ adenocarcinoma of the prostate: morphological comparison and biological significance. J Urol 1988;139:61-65.

3 Villers A, McNeal JE, Freiha FS, et al. Multiple cancers in the prostate. Morphologic features of clinically recognized versus incidental tumors. Cancer 1992;70: 2313-2318.

4 Stamey TA, McNeal JE, Yemoto CM, et al. Biological determinants of cancer progression in men with prostate cancer. JAMA 1999;281:1395-1400.
5 Griewe GL, Dean RC, Zhang W, et al. p53 Immunostaining guided laser capture microdissection (p53LCM) defines the presence of p53 gene mutations in focal regions of primary prostate cancer positive for p53 protein. Prostate Cancer Prostatic Dis 2003;6: 281-285.

6 Tomlins SA, Rhodes DR, Perner S, et al. Recurrent fusion of TMPRSS2 and ETS transcription factor genes in prostate cancer. Science 2005;310:644-648.

7 Petrovics G, Liu A, Shaheduzzaman S, et al. Frequent overexpression of ETS-related gene-1 (ERG1) in prostate cancer transcriptome. Oncogene 2005;24: 3847-3852.

8 Perner S, Demichelis F, Beroukhim R, et al. TMPRSS2:ERG fusion-associated deletions provide insight into the heterogeneity of prostate cancer. Cancer Res 2006;66:8337-8341.

9 Wang J, Cai Y, Ren C, et al. Expression of variant TMPRSS2/ERG fusion messenger RNAs is associated with aggressive prostate cancer. Cancer Res 2006;66: 8347-8351.

10 Iljin $\mathrm{K}$, Wolf M, Edgren $\mathrm{H}$, et al. TMPRSS2 fusions with oncogenic ETS factors in prostate cancer involve unbalanced genomic rearrangements and are associated with HDAC1 and epigenetic reprogramming. Cancer Res 2006;66:10242-10246.

11 Hermans KG, van Marion R, van Dekken $\mathrm{H}$, et al. TMPRSS2:ERG fusion by translocation or interstitial deletion is highly relevant in androgen-dependent prostate cancer, but is bypassed in late-stage androgen receptor-negative prostate cancer. Cancer Res 2006;66:10658-10663.

12 Demichelis F, Fall K, Perner S, et al. TMPRSS2:ERG gene fusion associated with lethal prostate cancer in a watchful waiting cohort. Oncogene 2007;26: 4596-4599.

13 Gao C, Petrovics G, Cullen J et al. Quantitative features of a common TMPRSS2-ERG fusion transcript in prostate cancer. J Urol 2007;177:94.

14 Oikawa T, Yamada T. Molecular biology of the Ets family of transcription factors. Gene 2003;303:11-34.

15 Qian J, Wollan P, Bostwick DG. The extent and multicentricity of high-grade prostatic intraepithelial neoplasia in clinically localized prostatic adenocarcinoma. Hum Pathol 1997;28:143-148.

16 Bastacky SI, Wojno KJ, Walsh PC, et al. Pathological features of hereditary prostate cancer. J Urol 1995;153: 987-992.

17 Greene DR, Egawa S, Neerhut G, et al. The distribution of residual cancer in radical prostatectomy specimens in stage A prostate cancer. J Urol 1991;145: 324-328.

18 Greene DR, Wheeler TM, Egawa S, et al. A comparison of the morphological features of cancer arising in the transition zone and in the peripheral zone of the prostate. J Urol 1991;146:1069-1076.

19 Kastendieck H. Correlations between atypical primary hyperplasia and carcinoma of the prostate. A histological study of 180 total prostatectomies. Pathol Res Pract 1980;169:366-387.

20 Marx J. Medicine. Fused genes may help explain the origins of prostate cancer. Science 2005;310:603.

21 Ahlers CM, Figg WD. ETS-TMPRSS2 fusion gene products in prostate cancer. Cancer Biol Ther 2006; 5:254-255.

22 Soller MJ, Isaksson M, Elfving P, et al. Confirmation of the high frequency of the TMPRSS2/ERG fusion gene 
in prostate cancer. Genes Chromosomes Cancer 2006; 45:717-719.

23 Yoshimoto M, Joshua AM, Chilton-Macneill S, et al. Three-color FISH analysis of TMPRSS2/ERG fusions in prostate cancer indicates that genomic microdeletion of chromosome 21 is associated with rearrangement. Neoplasia 2006;8:465-469.

24 Attard G, Clark J, Ambroisine L, et al. Duplication of the fusion of TMPRSS2 to ERG sequences identifies fatal human prostate cancer. Oncogene; e-pub ahead of print 16 July 2007.

25 Wang J, Cai Y, Ren C, et al. Expression of variant TMPRSS2/ERG fusion messenger RNAs is associated with aggressive prostate cancer. Cancer Res 2006;66: 8347-8351.

26 Rubin MA, Chinnaiyan AM. Bioinformatics approach leads to the discovery of the TMPRSS2:ETS gene fusion in prostate cancer. Lab Invest 2006;86:1099-1102.

27 Cerveira N, Ribeiro FR, Peixoto A, et al. TMPRSS2ERG gene fusion causing ERG overexpression precedes chromosome copy number changes in prostate carcinomas and paired HGPIN lesions. Neoplasia 2006;8: 826-832.

28 Laxman B, Tomlins SA, Mehra R, et al. Noninvasive detection of TMPRSS2:ERG fusion transcripts in the urine of men with prostate cancer. Neoplasia 2006;8:885-888.

29 Iljin K, Wolf M, Edgren $\mathrm{H}$, et al. TMPRSS2 fusions with oncogenic ETS factors in prostate cancer involve unbalanced genomic rearrangements and are associated with HDAC1 and epigenetic reprogramming. Cancer Res 2006;66:10242-10246.

30 Mehra R, Han B, Tomlins SA, et al. Heterogeneity of TMPRSS2 gene rearrangements in multifocal prostate adenocarcinoma: molecular evidence for an independent group of diseases. Cancer Res 2007;67: 7991-7995.

31 Nami RK, Sugar L, Wang Z, et al. Expression of TMPRSS2 ERG gene fusion in prostate cancer cells is an important prognostic factor for cancer progression. Cancer Biol Ther 2007;6:40-45.

32 Macaluso M, Giordano A. TMPRSS2:ERG gene fusion a new genetic marker for prostate cancer progression. Cancer Biol Ther 2007;6:46-47.

33 Liu W, Ewing CM, Chang BL, et al. Multiple genomic alterations on 21q22 predict various TMPRSS2/ERG fusion transcripts in human prostate cancers. Genes Chromosomes Cancer 2007;46:972-980.

34 Rajput AB, Miller MA, De Luca A, et al. Frequency of the TMPRSS2:ERG gene fusion is increased in moderate to poorly differentiated prostate cancers. J Clin Pathol 2007;60:1238-1243.

35 Mehra R, Tomlins SA, Shen R, et al. Comprehensive assessment of TMPRSS2 and ETS family gene aberrations in clinically localized prostate cancer. Mod Pathol 2007;20:538-544.

36 Lapointe J, Kim YH, Miller MA, et al. A variant TMPRSS2 isoform and ERG fusion product in prostate cancer with implications for molecular diagnosis. Mod Pathol 2007;20:467-473.

37 Mosquera JM, Perner S, Demichelis F, et al. Morphological features of TMPRSS2-ERG gene fusion prostate cancer. J Pathol 2007;212:91-101.

38 Winnes M, Lissbrant E, Damber JE, et al. Molecular genetic analyses of the TMPRSS2-ERG and TMPRSS2-ETV1 gene fusions in 50 cases of prostate cancer. Oncol Rep 2007;17:1033-1036.

39 Mertz KD, Setlur SR, Dhanasekaran SM, et al. Molecular characterization of TMPRSS2-ERG gene fusion in the NCI-H660 prostate cancer cell line: a new perspective for an old model. Neoplasia 2007;9:200-206.

40 Perner S, Mosquera JM, Demichelis F, et al. TMPRSS2ERG fusion prostate cancer: an early molecular event associated with invasion. Am J Surg Pathol 2007;31: 882-888.

41 Clark J, Merson S, Jhavar S, et al. Diversity of TMPRSS2-ERG fusion transcripts in the human prostate. Oncogene 2007;26:2667-2673

42 Emmert-Buck MR, Vocke CD, Pozzatti RO, et al. Allelic loss on chromosome 8p12-21 in microdissected prostatic intraepithelial neoplasia. Cancer Res 1995;55: 2959-2962.

43 Cheng L, Song SY, Pretlow TG, et al. Evidence of independent origin of multiple tumors from patients with prostate cancer. J Natl Cancer Inst 1998;90:233-237.

44 Leav I, McNeal JE, Ho SM, et al. Alpha-methylacylCoA racemase (P504S) expression in evolving carcinomas within benign prostatic hyperplasia and in cancers of the transition zone. Hum Pathol 2003;34: 228-233.

45 Bostwick DG, Qian J. High-grade prostatic intraepithelial neoplasia. Mod Pathol 2004;17:360-379. 